\title{
proDA: Probabilistic Dropout Analysis for Identifying Differentially Abundant Proteins in Label-Free Mass Spectrometry
}

\section{Constantin Ahlmann-Eltze}

University of Heidelberg and European Laboratory for Molecular Biology (EMBL)

Simon Anders ( $\sim$ sanders@fs.tum.de )

University of Heidelberg https://orcid.org/0000-0003-4868-1805

\section{Article}

Keywords:

Posted Date: June 23rd, 2020

DOI: https://doi.org/10.21203/rs.3.rs-36351/v1

License: (c) (i) This work is licensed under a Creative Commons Attribution 4.0 International License.

Read Full License 


\section{Abstract}

Protein mass spectrometry with label-free quantification (LFQ) is widely used for quantitative proteomics studies. Nevertheless, well-principled statistical inference procedures are still lacking, and most practitioners adopt methods from transcriptomics. These, however, cannot properly treat the principal complication of label-free proteomics, namely many non-randomly missing values. We present proDA, a method to perform statistical tests for differential abundance of proteins. It models missing values in an intensity-dependent probabilistic manner. proDA is based on linear models and thus suitable for complex experimental designs, and boosts statistical power for small sample sizes by using variance moderation. We show that the currently widely used methods based on ad hoc imputation schemes can report excessive false positives, and that proDA not only overcomes this serious issue but also offers high sensitivity. Thus, proDA fills a crucial gap in the toolbox of quantitative proteomics.

\section{Introduction}

Label-free quantification (LFQ) is a standard approach used in proteomics mass spectrometry (MS). Due to the similarity of this data type to expression microarray data, analysis methods from that field are commonly used for LFQ-MS. A major difference, however, is the presence of missing values in MS, but not in microarray data.

It is well established that missing values do not oc- cur entirely at random, but more often at low intensities $[1,2,3,4]$. The fraction of missing values varies by ex-perimental design, but it is not uncommon to have more than $50 \%$ missing values, especially in affinity purifica- tion experiments. This issue hence cannot simply be ignored but needs proper handling, and doing so is a cen- tral challenge in statistical analysis of LFQ data, e.g., for identifying proteins which are differentially abundant be- tween conditions. In the last years several method have been proposed to tackle this challenge, most of which rely on imputation, i.e., they simply replace missing val- ues with some number that is deemed realistic.

However, a fundamental problem with imputation is that it obscures the amount of available information: im- puted values will be considered as equally certain as ac- tually measured values by any downstream processing (identifying differentially abundant proteins, clustering, quality control). This can invalidate inferential conclu- sions due to underestimating statistical uncertainty or cause loss of statistical power. Therefore, we propose a probabilistic dropout model that explicitly describes the available information about the missing values.

Figure $1 \mathrm{~A}$ demonstrates that missingness carries information: observations in proteins with many missing values (red) have a lower intensity than observations in proteins with only one or no missing values (purple). In addition, Figure 1B illustrates that the ratio of these densities forms a curve with sigmoidal shape, clearly showing how the probability of a value being missing depends strongly on overall intensity. 
If sample size is limited, substantial gains in statis- tical power can be gained from using shrinkage estima- tion procedure for variance estimation ("variance mod- eration") [5]. This approach is widely used in transcrip-tomics data analysis, e.g., by the limma package [6]. The advantage of using limma or similar approaches for LFQ- MS has been advocated only rather recently (e.g., [7]). For example, the DEP package [8] performs imputation followed by a limma analysis to infer differentially abun- dant proteins. As stated above, the use of imputation may compromise the validity of limma's statistical infer- ence, and hence, the purpose of the present work is to adapt limma-style inference to account for values miss- ing not at random and so improve power and reliability of differential abundance analysis for LFQ-MS.

A typical analysis of a label-free tandem mass spec- trometry experiment consists of a number of steps. First, peaks in the MS1 need to be identified using the corre- sponding MS2 spectra. Second, the MS1 peaks need to be quantified. In the literature, two approaches are pop- ular for this tasks: spectral counting and peak area inte- gration [9]. Abundant peptides are more often recorded by the MS2, thus the number of MS2 spectra associated with a peptide can be used as a proxy for its abundance [10]. Alternatively, more abundant proteins cause larger peaks in the MS1, thus a second approach is to integrate the peak area of a peptide [11, 12]. Subsequent compar- isons of the methods concluded that peak area based methods perform better than spectral counting $[13,14]$. Consequently, we will only focus on methods that handle continuous intensities.

The third important step is the aggregation of the peptide level information to protein information. Traditionally, the peptide intensities are aggregated to protein intensities and then in a separated step the differential abundance is calculated for each protein. One popu- lar method, that is directly integrated in the MaxQuant platform [15], is called MaxLFQ that uses delayed nor- malization based on the peptide ratios [16]. Alterna- tive methods include summing up the peptide intensi- ties, taking the average of the top three peptides [17], selecting a reference peptide to calculate the protein in- tensity [18], averaging the ratios [19], or using relative abundances while taking into account shared peptides [20]. More recently, some methods have been published that directly try to combine both steps to gain more power. The result of all those steps is a table with in- tensities for each protein and sample. The values in this table are commonly on a log2 scale in order to account for the mean-variance relationship of the raw data (Supplementary Figure S1).

Several methods have been published in the last years that use those protein intensities to calculate differ- ential abundance. Perseus [21] is a platform with a graphical user interface, developed by the same group as MaxQuant, which provides functionality to normal- ize the data, impute missing values, identify significant proteins using a t-test and visualize the results. For multiple testing correction, Perseus offers two options: either the Benjamini-Hochberg procedure [22] or signif- icance analysis of microarrays (SAM), a permutation- based correction originally described in Ref. [23]. As already mentioned, DEP [8] is an $\mathrm{R}$ package that pro- vides a similar set of functionalities, but uses the more powerful variance moderated t-test to identify significant proteins using the $\mathrm{R}$ package limma [6, 24]. For multi- ple testing correction, DEP uses by default the methods in the fdrtool package [25]. In order to handle miss- ing values, it provides an interface to a large number of imputation methods from the MSnbase R package 
[26]. In contrast, Perseus only provides two imputation meth- ods, which either replace the missing values with a small deterministic value (MinDet) or with random values jit- tered around that small value (MinProb). DAPAR and ProStaR [27] are complementary software tools where DAPAR is an R-package that is similar to DEP, but has additional imputation methods based on the imp4p package [28]. ProStaR internally uses DAPAR and pro- vides a web-based graphical user interface to make the software more approachable to newcomers.

Approaches that work without imputation are limited so far. one approach is the "empirical Bayesian random censoring threshold" (EBRCT) model, which avoids im- putation by integrating over the inferred intensity dis- tribution for missing values [29]. However, it cannot handle the case if a protein is completely missing in one condition. This can actually be problematic because in an affinity purification experiment those proteins might actually be the ones that we care about the most. An- other tool that follows a similar idea is QPROT [4]; a command line tool that uses empirical Bayesian priors and integrates out the position of missing values using a cumulative normal distribution below a hard limit of detection.

Lastly, Triqler [31] is a tool that directly works on the peptide level instead of the protein level. This has the advantage that it can incorporate additional uncertainty due to the integration of multiple peptides to one protein intensity. It is a command line tool written in Python that fits an empirical Bayesian model integrating out the uncertainty for missing peptide quantifications.

Here, we present proDA (inference of protein $d$ ifferential abundance by probabilistic $d$ ropout analysis), a novel method for infering differential abun- dance that makes full use of the information inherent in missingness. proDA models the random process resulting in missingness and so avoids the problems discussed above that are inherent to imputation-based inference.

In the following section, we explain the intuition be- hind proDA and how it differs from the existing tools. In the third section, we use a spike-in and a semi-synthetic dataset to perform benchmarks. We show that many exiting methods have either low statistical power, or seri- ous deficiancies in controlling false discovery rate (FDR), i.e., they refer too many false positives, and that hence the need for a general, powerful and statistically reliable inference method is unmet. We show that proDA offers strong performance with reliable FDR control, and thus is suitable to fill this crucial gap in existing methodology. In the fourth section, we demonstrate proDA in an application setting, by analyzing a real dataset studying ubiquitination. We close with a conclusion.

\section{Approach}

The core of our idea is to combine the sigmoidal dropout curve for missing values with the information from the observed values. Figure 2 gives a conceptual overview of our approach. Our method works on the protein- level intensities, because this makes it compatible with a variety of different intensity aggregation methods, easier to use, and, as we will see later, the performance benefits from working on the peptide level are unclear. 
All the mathematical details of our method are described in the Appendix, where we develop the approach for full linear models. Here, in the main text, we aim to provide a more intuitive explanation. We will first discuss the simple setting of an experiment with only a single condition with 3 replicates, and afterwards discuss inference of differential abundance between two conditions.

We assume that, within one condition, there is one expected value for each protein, the population average, i.e., the mean value we would get if we averaged over infinitely many replicate samples. The abundances in our 3 replicates scatter around this unknown "true" mean value, and our goal is to infer a posterior distribution that contains the true mean and captures our uncertainty about its location. In case of no missing values, such a posterior takes the shape of the t distribution, which is the basis for the well known Student's t test (green posterior in Figure 2). Missing values cause these posteriors to become skewed, wider, and their mode (peak) to shift to the left of the average of the observed values (because the missing values are likely lower than the observed ones); see orange posterior in Figure 2. Even with no observed values, we can infer a posterior (purple posterior in the figure): its left flank follows the location prior, i.e., the distribution of values we actually expect in our data, and its right flank follows the dropout curve, because higher values would have likely been observed.

Hence, our approach first estimates from the data for all proteins the shape of the dropout probability curve and of the location prior. It then uses this information to infer for each protein an approximate posterior for its mean, with the necessary shift in mode location and widening due to the additional uncertainty from any missing values. We approximate the skewed posteriors with a symmetric approximation (dashed lines in the figure) that follows the right flank. (See Appendix, Section "Variance of the Coefficient Estimates" for details.)

The process of parameter estimation involves so-called shrinkage estimation (or moderation), which shares information across proteins in order to improve variance estimation (as originally proposed in Ref. [5] and also used in limma [6]). Furthermore, we apply shrinkage estimation not only to the variance but also to the location, as this enables us to handle the edge case of all observation missing in one condition.

To test if a protein is differentially abundant between two conditions, we compare the approximate posteriors inferred for the two conditions and calculate a $p$-value for the null hypothesis of both true means being equal. We do this using linear models, which allows for accommodating known covariates and complex experimental designs, in the same manner as limma offers for transcriptomics experiments.

\section{Validation And Comparison}

We validated our approach and compared it with the existing methods discussed in the introduction. To this end, we used a dataset by de Graaf et al. [32], who analysed phosphorylation dynamics of Jurkat T cells over 6 time points using affinity purification. We only use the first time point, for which there are 18 samples, 3 biological replicates with 3 technical replicates each, which were measured in two separate mass spectrometry runs. As all these samples are in the same condition, we a pri-ori do not expect any 
differences between the samples. We then introduce the changes ourselves so that we have a known ground truth. This has the additional advantage that we can vary the number of compared samples and the fraction of truly changed proteins to see how those affect the results. As a first test, however, we run our methods, as well as a variety of other methods, on the data as is, without any real differences between conditions, in order to check whether any of the tools might nevertheless falsely report statistical differences.

\section{Null comparison}

Supplementary Figure S2 shows a heatmap of the data. There are many missing values (49\%), which helps us to assess their impact on the different methods. In a typical affinity purification experiment, it is not unusual to have only three replicates per condition. So, we chose six samples and divided them into two synthetic conditions, ensuring that both contain a mix of different biological replicates, so that there is no signal in the null dataset (row marked " $3 \mathrm{v} 3$ " in Supplementary Figure S2).

We compare the 7 methods discussed in the introduction (Perseus, DEP, DAPAR, QPROT, EBRCT, Triqler, and proDA), running each tool with their default set- tings, except for the multiple testing correction, where we stick to the Benjamini-Hochberg method wherever possible, in order to make the results more comparable. DEP offers a range of different imputation methods; we chose to test it with five typical ones: Zero, MinDet, MinProb, KNN, and QRLIC. For DAPAR we used the structured least squares algorithm from the imp4p package [28, 33]. We ran QPROT with 2000 burn-in and 10,000 sampling iterations. For EBRCT, we have to filter out the proteins where for one condition all proteins are missing, because it cannot handle that edge case. Lastly, Triqler was a little more challenging to use, because it needs the data in a specific format that includes the decoy matches to calculate the FDR. For Triqler specifically, following the advice of the Triqler authors, we re-ran the MaxQuant quantification, with PSM and protein detection FDR set to $100 \%$ and matching between runs turned off, then converted the evidence file to Triqler input, skipping the normalization. We ran Triqler version 0.3.1 with the fold change eval setting changed to 0 . It should be kept in mind that this means that Triqler was run on a slightly different data set without the same normalization and that thus the performance results are not one-to-one comparable.

Figure 3 shows the results: proDA correctly detects no significant proteins, as do most tested methods. The DEP method combined with zero imputation proves to be problematic, as are QPROT and EBRCT. DAPAR performance worse with increasing number of samples (" $4 \mathrm{v} 4$ " and "6v6" Supplementary Figure S3 and S4). Triqler (which is not included in the plots, because it does not calculate classical p-values) detects an unacceptably large number of false positives, which is why we will exclude it from the subsequent analyses.

\section{Semi-synthetic dataset}

We now introduce the artificial changes so that we have a known ground truth. We select $20 \%$ of all proteins and randomly shuffle those rows, but only in the first condition. This creates a realistic dataset where we know which proteins differ between condition one and two. The more common approach, 
where a selected number of proteins are shifted by a fixed effect size, is not applicable here, because shifting the mean of a protein would also imply a different probability for missing observations. Unfortunately, this idea can not be consistently applied to peptide level data, which is why we will have to leave Triqler out of the following benchmark.

We compared the other 6 method (Perseus, DEP, DA- PAR, QPROT, EBRCT, and proDA), ran them with the same settings described in the previous section.

Figure $4 A$ and $B$ show the performance of the tools: in this test, DEP with most imputation methods (except\$ zero imputation) and proDA succeed in controlling the FDR. EBRCT, QPROT, and DAPAR fail to control it. For those methods that passed the FDR control requirement, we can again ask which has most inferential power. Figure 4D shows the number of true positives that each method recovered depending on the desired FDR. proDA performs well in this test. Its actual FDR always stays below the desired FDR and at $10 \%$ desired FDR, it recovers $65 \%$ more true positives than the second best approach, DEP with MinDet imputation.

The performance of DEP depended on the imputation method that is used. Zero imputation is problematic, as can be seen in this example, because it fails to control the FDR at small values. The best imputation methods are MinDet and MinProb, which perform nearly identical. Perseus with the MinProb imputation recovers fewer true positives than DEP, which is expected, because it uses the classical t-test and not the variance moderated version provided by limma.

In Supplementary Figure S5, we further distinguish the calibration and performance by the number of actually observed values in condition one and two. This shows that the failure of QPROT to control the FDR is because it has too many false positives specifically for proteins with zero against one observation. The opposite pattern is observed for EBRCT, that has too many false positive detections if a protein is observed in all conditions or mising in just one. proDA is more powerful than the other methods, because it shows consistently good performance across comparisons and in particular if only one or two observations are missing. DEP with the zero imputation method always identifies all proteins fully observed in one condition and completely missing in the other as significant. In many cases this is correct, but as this does not depend on the desired FDR, this can lead to an actual FDR that is too large if the user specifies a small FDR.

In Supplementary Figure S6-S8, we show that we mostly get consistent results even if we change the number of compared samples ( 3 vs 3,4 vs 4 , and 6 vs 6 ) and also if we change the percentage of true positive proteins $(5 \%, 10 \%, 20 \%$, and $30 \%)$. proDA reliably controls the FDR, except in two circumstances where the noise from the small number of changed genes (5\% in Supplemen- tary Figure S7A and Supplementary Figure S8A) causes a small violation of the FDR control. It also detects more true positives in most of the comparison than the other tools, especially whenever there are many missing values. 


\section{Application}

After demonstrating that only few tools, including proDA, control the FDR reliably, and that proDA is able to recover the largest number of changed proteins, we applied it to analyze a data set on the interaction landscape of ubiquitin [34]. In this example, we do not know the ground truth, but show that we recover proteins that biologically make sense. In the original publication the authors analyzed the data set using Perseus, later they presented the DEP R package for analyzing such data sets [8]. We will re-run the analysis that Zhang et al. describe with proDA.

Ubiquitin is a small protein that plays an important role in many different signaling pathways. There are three different kinds of ubiquitination: mono- ubiquitination, multi-mono-ubiquitination and polyubiquitination. Poly-ubiquitination is further distinguished by the linkage between the donor and the acceptor ubiquitin. The donor is linked with its $C$ terminus to any of the seven lysines (K6, K11, K27, K29, $\mathrm{K} 33, \mathrm{~K} 48, \mathrm{~K} 63$ ) or the terminal methionine (M1) of the acceptor. Zhang et al. studied the recognition of those eight linkages and mono-ubiquitin by ubiquitin binding proteins. For this, they developed a new technique called ubiquitin interactor affinity enrichment-mass spectrometry (UbIA-MS) [8].

They run an enrichment experiment for each of the eight ubiquitin linkages plus one condition with monoubiquitin (Mono) and one empty control condition (ctrl). Each condition was measured in triplicates. To determine which proteins bind (directly or indirectly) to any of the ubiquitin linkages, we always compare the intensity for each protein to the corresponding intensity in the control group.

Figure 5 shows the results of the analysis with proDA. Figure $5 \mathrm{~A}$ compares the total number of significant inter-actors at a nominal FDR of $10 \%$, filtering out all proteins that had higher intensity in the control condition than in the ubiquitin condition. Figure 5B further stratifies the data from panel A. It not just describes how many pro- teins bind to a linkage, but also how many proteins bind to a specific combination of linkages. We can see that a majority interacts significantly with with all ubiquitins, but there are also proteins showing significant interactions only for specific linkages.

Figure 5D demonstrates that proDA has not just recovered many interactors, but proteins related to gene ontology sets relevant for ubiquitination [35, 36, 37]. In addition to the 9 ubiquitination conditions, here we also list the results of conducting an $\mathrm{F}$ test to identify all proteins that differ in any condition, as an example for the ability of proDA to perform missing value aware ANOVA.

\section{Distances}

A commonly used approach for sample quality control is to calculate some measure of similarity for all pairs of samples, in order to check whether replicate samples appear more similar than samples from different conditions.

Typically, Euclidean distance is used, e.g. in the ubiquitilation study by Zhang et al. [8] who use MinProb imputation before Euclidean distance is calculated. Fig- ure $5 \mathrm{C}$ shows the outcome of this procedure for 
the ubiquitin data. Differences in the shape of the dropout probability curve can strongly influence a distance calculated in this manner. Based on the proDA model, we developed an approach to calculate Euclidean distance in a probabilistic manner without the need for imputation in order to reduce the effect of differences in dropout probabilities (Appendix, section "Distances")). In fact, our distance calculation is able to recover the triplet structure of the data set, while the MinProb imputation based distances do not (Figure 5C).

\section{Conclusion}

In this paper, we have presented our R package proDA for identifying proteins that are differentially abundant in label-free mass spectrometry data sets. The main challenge for analyzing label-free mass spectrometry data are the large number of missing values. We suggest to handle them using a probabilistic dropout model combined with empirical Bayesian priors to combine the available information from observed and missing values.

In the performance comparison with existing tools with and without any true positives, we saw that some method produce a lot of false positives. In particular, Triqler had difficulties on the null-comparison. This could be due to the challenges of balancing the different levels of uncertainty from the missing values and the observations on the peptide level.

On the semi-synthetic data set, we saw that proDA recovers more true positives, while controlling the false discovery rate. We showed that imputation can be problematic because it either leads to a loss of power or worse to not controlling the false discovery rate. The improved sensitivity of proDA comes at the prize of a somewhat increased run time. Whereas DEP, DAPAR, and Perseus finish within seconds, our model might need one or two minutes to calculate a result. In the end, we believe the increased computational demands are justified, because the analysis run time is still fast enough for interactive use.

In addition, our tool can handle any design specified as a linear model. This has the advantage that one can not just fit two condition comparisons, but also time series data, nested data with patient and treatment specific effects, and account for known covariates in the model. Besides proDA, this is only supported by DEP although this is standard for transcriptomic tools.

In conclusion, we have demonstrated that imputation can be problematic and that properly modelling the uncertainty posed by missing values boosts power.

\section{Declarations}

\section{Availability}

Software: The proDA method is implemented as an open-source R package. Documentation, installation instructions and download links are provided at the BioConductor package repository https://www.bioconductor.org/packages/proDA/. 
License: proDA is made available as open-source software under the GNU General Public License, version 3 or later.

Source code for software: Full source code for the software is available on GitHub, at https://github.com/const-ae/proDA

Source code for benchmarks and example analyses: The full R code to produce all the figures and all the benchmark and example application results reported here is available at github.com/const-ae/proDAPaper as R notebooks. The example data used was downloaded using the accessions given in Refs. [32] and [34]; details are given in the R notebooks.

\section{Funding}

This work has been supported by the Deutsche Forschungsgemeinschaft (DFG) Collaborative Research Centre SFB 1036.

\section{References}

(Note: The symbol “ C? ” marks clickable DOI hyperlinks.)

1. Lazar, C., Gatto, L., Ferro, , Bruley, C. \& Burger, T. Ac- counting for the multiple natures of missing values in label-free quantitative proteomics data sets to compare imputa- tion strategies. Journal of Proteome Research 15, 1116-1125 (2016). C?

2. Välikangas, , Suomi, T. \& Elo, L. L. A comprehensive evaluation of popular proteomics software workflows for label- free proteome quantification and imputation. Briefings in Bioinformatics 1, 1-12 (2017). C?

3. Ooijen, M. V. et al. Identification of differentially expressed peptides in high-throughput proteomics data. Briefings in Bioinformatics 1, 1-11 (2017). C?

4. Choi, H., Kim, S., Fermin, , Tsou, C. C. \& Nesvizhskii, A. I. QPROT: Statistical method for testing differential expression using protein-level intensity data in label-free quantitative proteomics. Journal of Proteomics 129, 121-126 (2015). C?

5. Lönnstedt, \& Speed, T. Replicated microarray data. Sta- tistical Sinica 12, 31-46 (2002).

6. Smyth, G. K. Linear models and empirical Bayes methods for assessing differential expression in microarray experiments. Statistical Applications in Genetics and Molecular Biology 3, 1-26 (2004). C?

7. Kammers, K., Cole, R. N., Tiengwe, C. \& Ruczinski, I. De- tecting significant changes in protein abundance. EuPA Open Proteomics 7, 11-19 (2015). C?

8. Zhang, X. et al. Proteome-wide identification of ubiquitin interactions using UbIA-MS. Nature Protocols 13, 530-550 (2018). C?

9. Wong, W. H. \& Cagney, G. An overview of label-free quantitation methods in proteomics by mass spectrometry. In Proteome Bioinformatics, 273-283 (Springer, 2010). C? 
10. Liu, H., Sadygov, R. G. \& Yates, R. A model for ran- dom sampling and estimation of relative protein abundance in shotgun proteomics. Analytical Chemistry 76, 4193-4201 (2004). C?

11. Bondarenko, V., Chelius, D. \& Shaler, T. A. Identification and relative quantitation of protein mixtures by enzymatic di- gestion followed by capillary reversed-phase liquid chromatog- raphy - Tandem mass spectrometry. Analytical Chemistry 74, 4741-4749 (2002). C?

12. Chelius, D. \& Bondarenko, V. Quantitative profiling of proteins in complex mixtures using liquid chromatography and mass spectrometry. Journal of Proteome Research 1, 317-323 (2002). C?

13. Grossmann, J. et al. Implementation and evaluation of rela- tive and absolute quantification in shotgun proteomics with label-free methods. Journal of Proteomics 73, 1740-1746 (2010). C?

14. Dowle, A. A., Wilson, \& Thomas, J. R. Comparing the diagnostic classification accuracy of iTRAQ, peak-area, spectral-counting, and emPAI methods for relative quantifi-cation in expression proteomics. Journal of Proteome Re- search 15, 3550-3562 (2016). C?

15. Cox, J. \& Mann, M. MaxQuant enables high peptide identifi- cation rates, individualized p.p.b.-range mass accuracies and proteome-wide protein quantification. Nature Biotechnology 26, 1367-1372 (2008). C?

16. Cox, J. et al. Accurate proteome-wide label-free quantifica- tion by delayed normalization and maximal peptide ratio ex- traction, termed MaxLFQ. Molecular \& Cellular Proteomics 13, 2513-2526 (2014). C?

17. Choi, H., Glatter, T., Gstaiger, M. \& Nesvizhskii, A. I. SAINT-MS1: Protein-protein interaction scoring using label- free intensity data in affinity purification-mass spectrometry experiments. Journal of Proteome Research 11, 2619-2624 (2012). C?

18. Polpitiya, A. D. et al. DAnTE : a statistical tool for quanti- tative analysis of -omics data. Bioinformatics (Oxford, Eng- land) 24, 1556-1558 (2008). C?

19. Bantscheff, M., Schirle, M., Sweetman, G., Rick, J. \& Kuster, Quantitative mass spectrometry in proteomics: A critical review. Analytical and Bioanalytical Chemistry 389, 1017- 1031 (2007). C?

20. Dost, B. et al. Accurate mass spectrometry based protein quantification via shared peptides. Journal of Computational Biology 19, 337-348 (2012). C?

21. Tyanova, et al. The Perseus computational platform for comprehensive analysis of (prote)omics data. Nature Methods 13, 731-740 (2016). C?

22. Benjamini \& Controlling the false discovery rate: a practical and powerful approach to multiple testing. Journal of the Royal Statistical Society, Series B 57, 289-300 (1995).

23. Tusher, G., Tibshirani, R. \& Chu, G. Significance analy- sis of microarrays applied to the ionizing radiation response. PNAS 98 (2001). C?

24. Ritchie, M. E. et al. limma powers differential expression analyses for RNA-sequencing and microarray studies. Nucleic Acids Research 43, e47-e47 (2015). C?

25. Strimmer, K. fdrtool: A versatile R package for estimating local and tail area-based false discovery rates. Bioinformatics 24, 1461-1462 (2008). C? 
26. Gatto, \& Lilley, K. MSnbase - an R/Bioconductor package for isobaric tagged mass spectrometry data visualization, pro- cessing and quantitation. Bioinformatics 28, 288-289 (2012).C?

27. Wieczorek, S. et al. DAPAR \& ProStaR: Software to per-form statistical analyses in quantitative discovery proteomics. Bioinformatics $33,135-136$ (2017). C?

28. Gianetto, Q. G. imp4p: Imputation for Proteomics (2018). URL https://cran.rproject.org/package $=$ imp4p.

29. Koopmans, F., Cornelisse, L. N., Heskes, T. \& Dijkstra, T. M. Empirical Bayesian random censoring threshold model im- proves detection of differentially abundant proteins. Journal of Proteome Research 13, 3871-3880 (2014). C?

30. Carpenter, B. et al. Stan: A probabilistic programming lan- guage. Journal of Statistical Software 76 (2017). C?

31. The, M \& Käll, L. Integrated identification and quantifica- tion error probabilities for shotgun proteomics. Molecular \& Cellular Proteomics 18, 561-570 (2019). C?

32. de Graaf, L., Giansanti, P., Altelaar, A. F. M. \& Heck, A. R. Single-step enrichment by Ti4+-IMAC and label-free quantitation enables in-depth monitoring of phosphorylation dynamics with high reproducibility and temporal resolution. Molecular \& Cellular Proteomics 13, 2426-2434 (2014). C?

33. Bo, T. H. LSimpute: accurate estimation of missing values in microarray data with least squares methods. Nucleic Acids Research 32, 34e-34 (2004). C?

34. Zhang, X. et al. An interaction landscape of ubiquitin signal-ing. Molecular Cell 65, 941-955.e8 (2017). C?

35. Ashburner, M. et al. Gene ontology: tool for the unification of biology. The Gene Ontology Consortium. Nature Genetics 25, 25-9 (2000). C?

36. Carbon, S. et al. The Gene Ontology Resource: 20 years and still GOing strong. Nucleic Acids Research 47, D330-D338 (2019). C?

37. Yu, G., Wang, L.-G., Han, Y. \& He, Q.-Y. clusterProfiler: an R package for comparing biological themes among gene clusters. Omics: a Journal of Integrative Biology 16, 284- 287 (2012). C?

38. Dunn, K. \& Smyth, G. K. Generalized Linear Models with Examples in R (Springer, 2018).

39. Ellison, B. E. Two theorems for inferences about the normal distribution with applications in acceptance sampling. Jour- nal of the American Statistical Association 59, 89-95 (1964).C?

40. Zacks, S. Parametric Statistical Inference: Basic Theory and Modern Approaches (Pergamon Press, 1981).

41. Gay, M. Usage summary for selected optimization rou- tines. Tech. Rep. 153, AT\&T Bell Laboratories, Murray Hill, NJ (1990).

42. Broyden, C. G. The convergence of a class of double-rank minimization algorithms. IMA Journal of Applied Mathe- matics 6, 76-90 (1970). C?

43. Fletcher, A new approach to variable metric algorithms. The Computer Journal 13, 317-322 (1970). C? 
44. Goldfarb, D. A family of variable-metric methods derived by variational means. Mathematics of Computation 24, 23-23 (1970). C?

45. Shanno, D. F. Conditioning of quasi-Newton methods for function minimization. Mathematics of Computation 24, 647-647 (1970). C?

46. Efron, B. \& Morris, C. Data analysis using Stein's estimator and its generalizations. Journal of the American Statistical Association 70, 311-319 (1975).

47. Mathai, A. \& Provost, S. Quadratic Forms in Random Vari- ables (CRC Press, 1992).

\section{Figures}
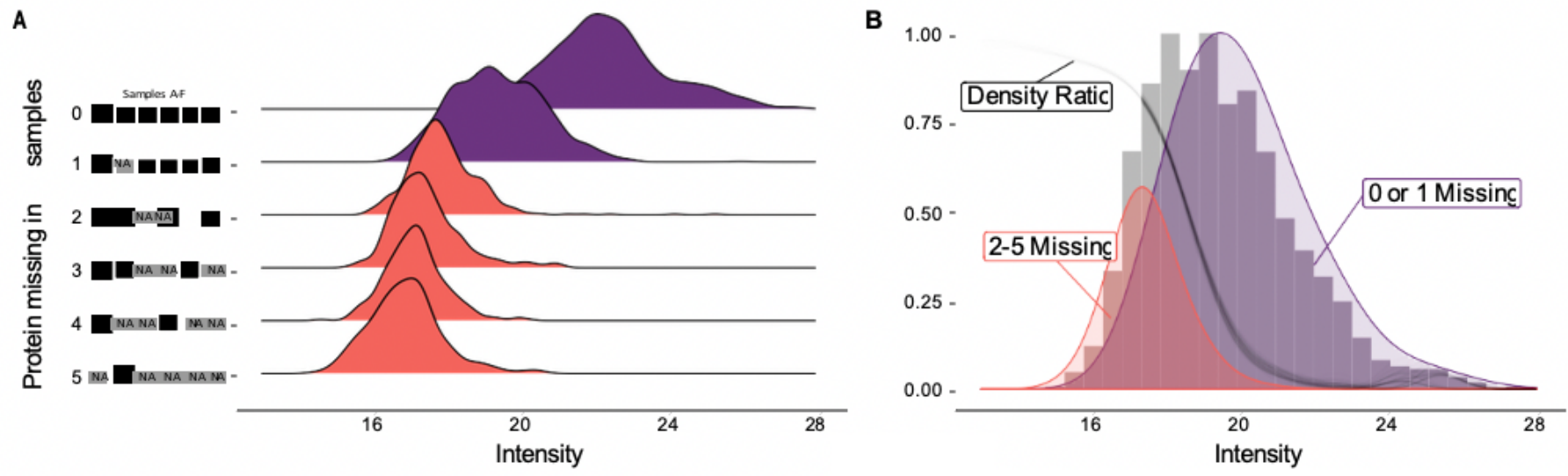

\section{Figure 1}

Missingness in label-free mass spectrometry is informative. (A) Intensity distribution for six replicates of the de Graaf data set. Shown is a ridgeline density plot of all the observed intensity values. They have been stratified by the number of samples in which the protein's value was missing. The height of the individual densities is normalized per stratum. Panel (B) shows, in gray, a histogram of all the intensities. Overlayed are densities combining either the values from proteins with at most one missing value (purple) or with more then one missing value (red). The ratio of these two densities (gray line) has sigmoidal shape. The density ratio has been bootstrapped 100 times to show its sampling distribution. 


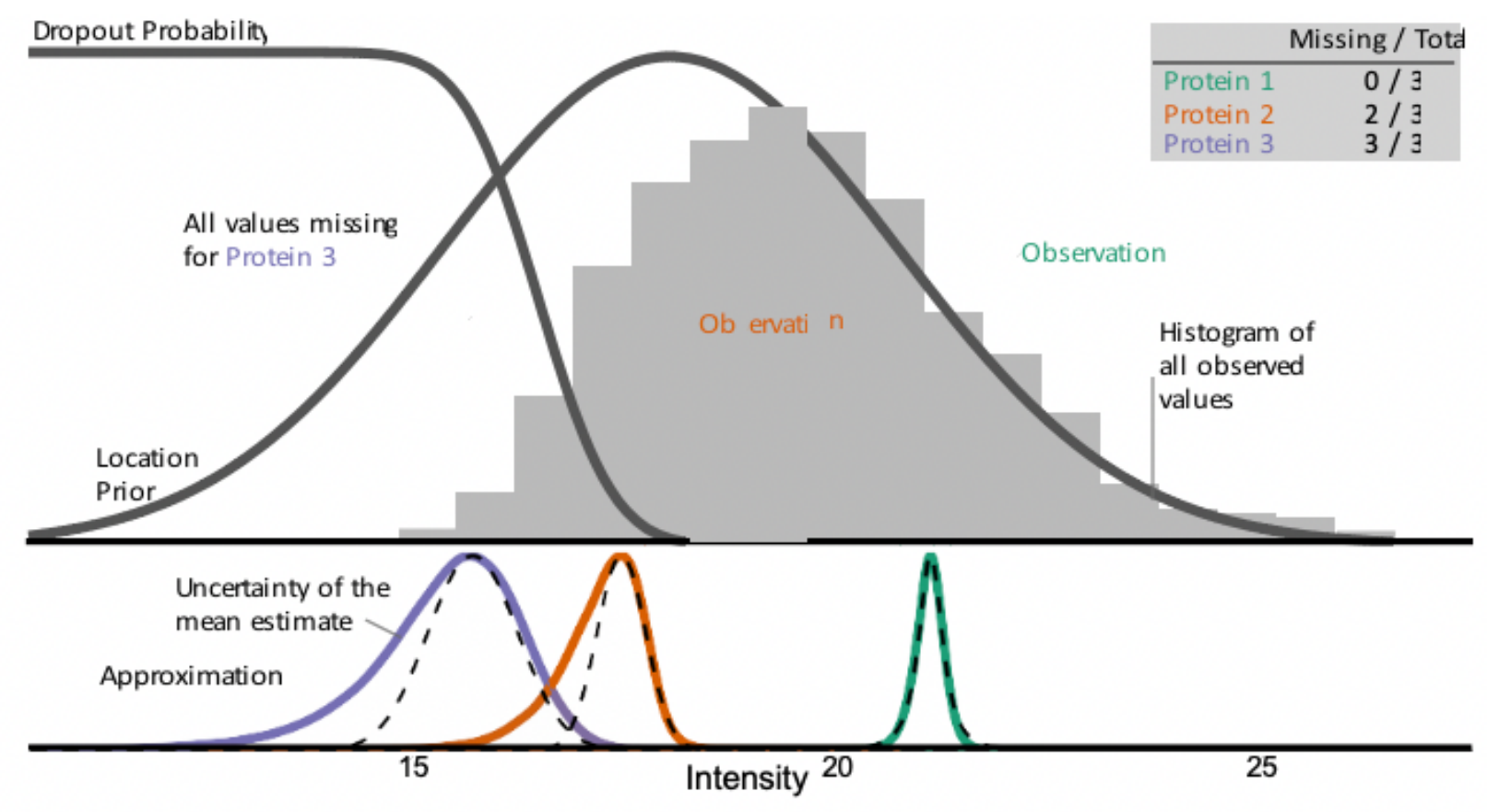

Figure 2

Intuition behind the probabilistic dropout model. We assume that the real intensity values approximately follow a normal distribution ("location prior"). The probability of not observing one of these values ("dropout probability") is high for low intensity and low for high intensity. Hence, the distribution of the actually observed values (gray histogram) is skewed, with values missing in its left flank. The vertical lines indicate the observed intensities for three hypothetical proteins: Protein 1 (green) has complete observations, protein 2 (orange) has 2 missing values, and protein 3 (purple) has only missing values. The lower panel shows the inferred posterior probability distribution for the means for proteins 1, 2, and 3 (calculated using Stan [30]). The dashed lines show the symmetric approximation to these that we use for efficient inference. 

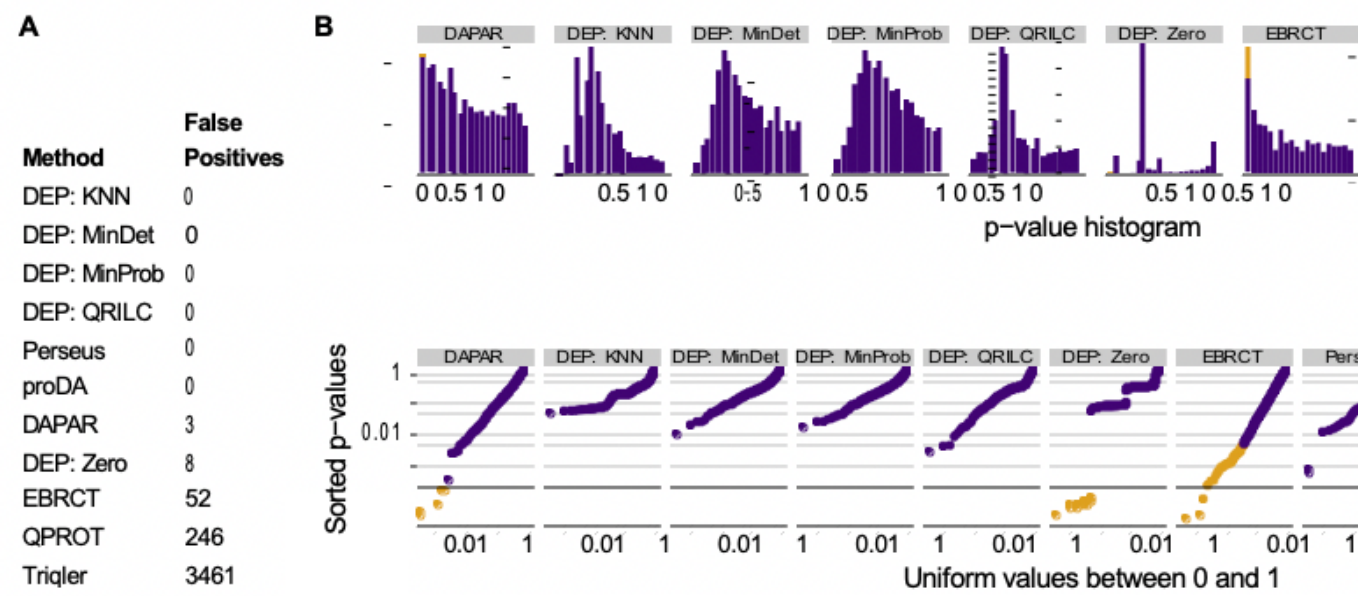

$\mathrm{p}$-value histogram
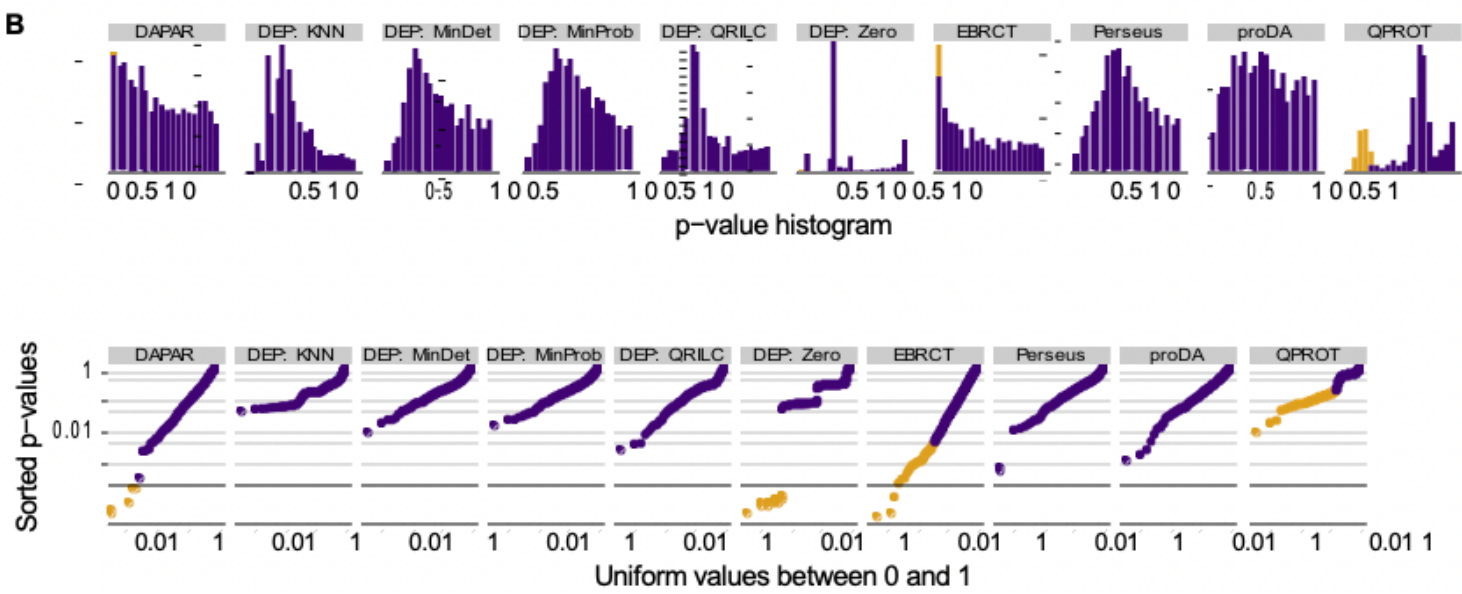

adjusted $p$-value $<0.1$ adjusted $p$-value $>0.1$

\section{Figure 3}

Comparison of the various tools' ability to avoid false-positives: We have compared three replicates in the de Graaf dataset with three further replicates, i.e., with no differences, and would hence expect uniform $p$ values and no detection of differentially expressed proteins after Benjamini-Hochberg adjustment. (A) Table of the number of falsely detected positives. (B) Histogram of $p$ values. Ideally, these should be uniform. Each tick on the $y$ - axis indicates 50 proteins. (C) Double-logarithmic QQ plots of $p$ values; orange colour indicates proteins deemed significant at 10\% FDR after BH adjustment. The QQ plots show the same data as the histograms but make it easier to judge uniformity for very small $p$ values. Triqler is absent in the histogram and double QQ plot, because it does not calculate p-values, but directly calculates the FDR.

A

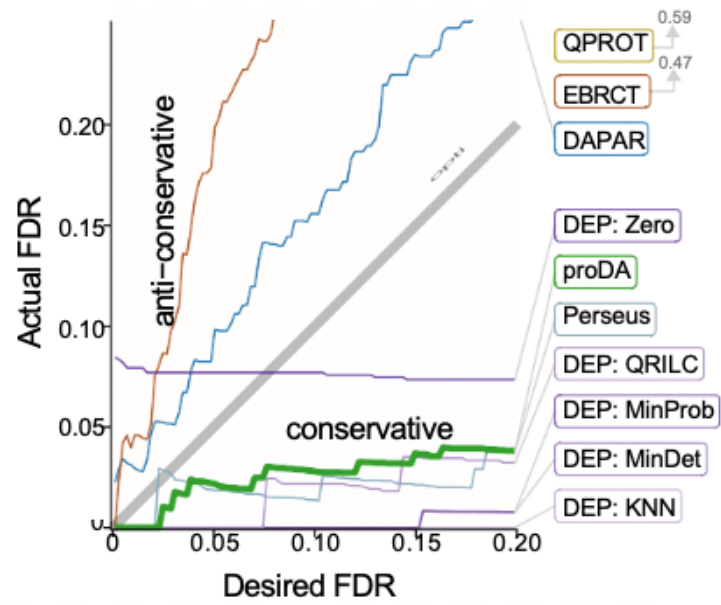

B

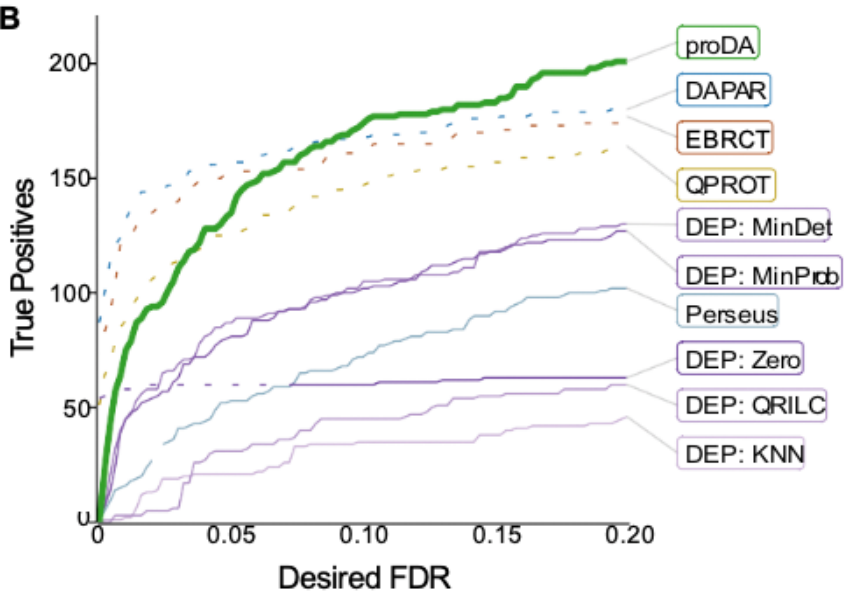




\section{Figure 4}

Performance comparison on the semi-synthetic data by de Graaf with three replicates and $20 \%$ changed proteins. A) Comparison of the user specified FDR (cut-off on BH-adjusted $p$ value) with the FDR that is actually achieved by the tool according to the ground truth. (The line for QPROT is not shown as it is lierally off the scale.) B) Plot showing how many actually changed proteins (true positives) each method identified at a specified FDR level. The methods shown as dotted lines should be considered "disqualified" as they that failed to control the FDR in panel A.

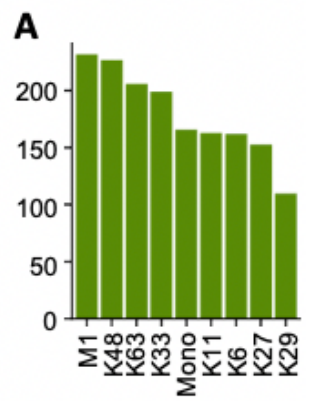

B

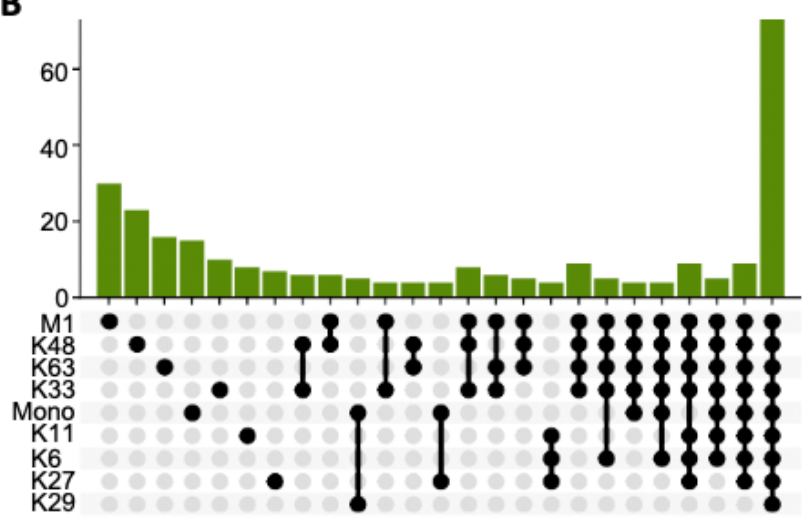

D

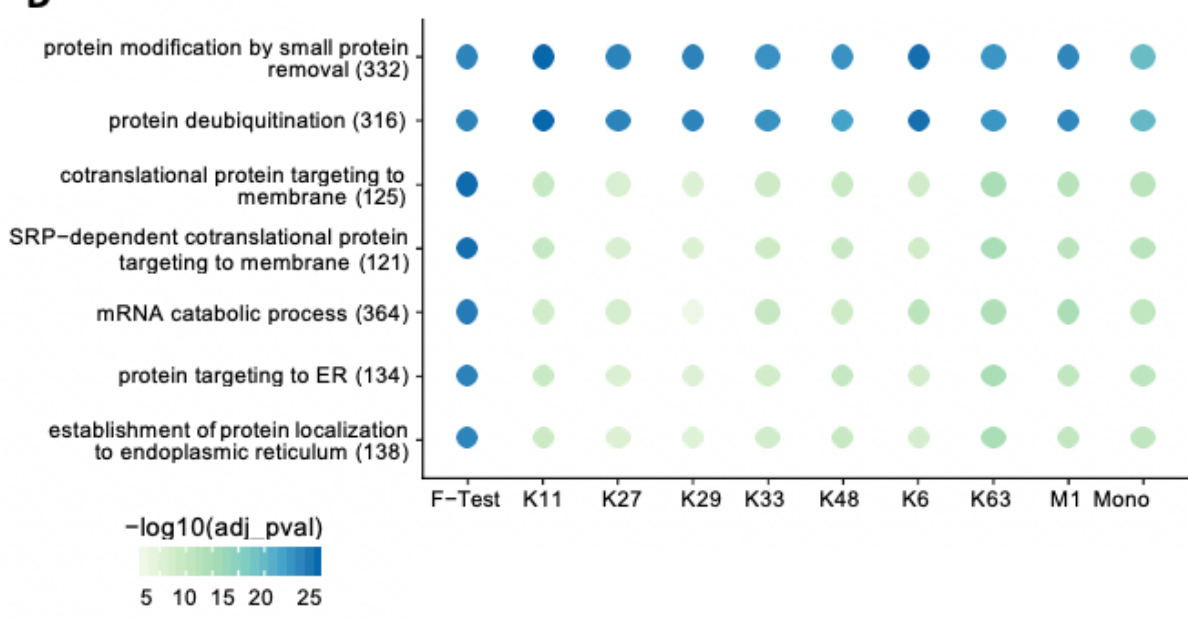

C
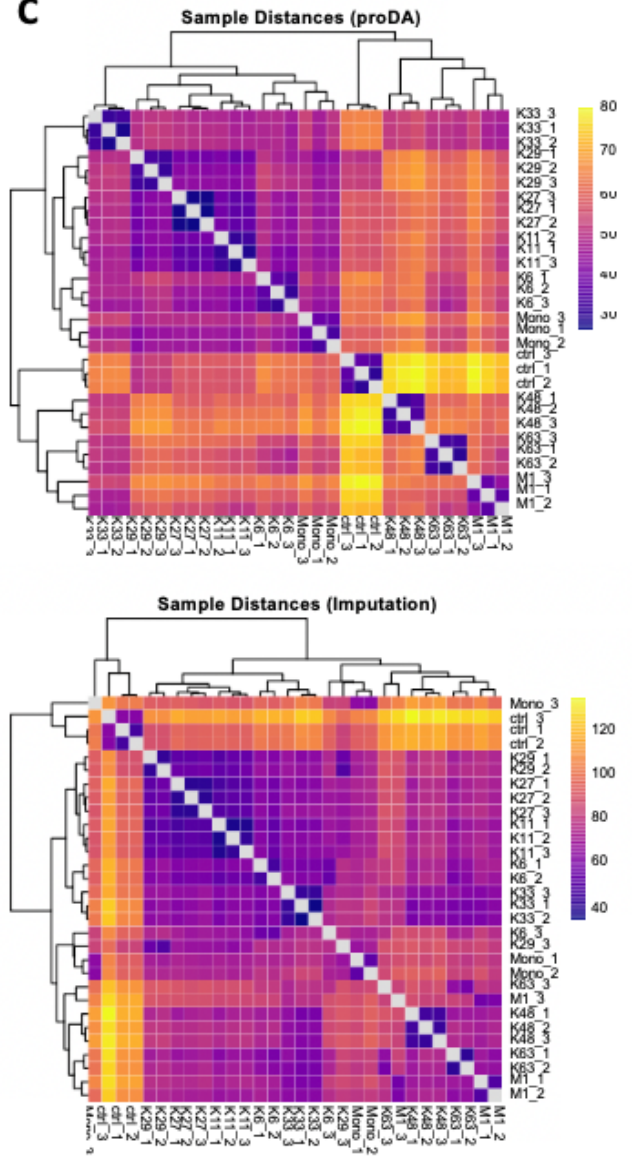

\section{Figure 5}

Performance comparison on the semi-synthetic data by de Graaf with three replicates and $20 \%$ changed proteins. A) Comparison of the user specified FDR (cut-off on BH-adjusted $p$ value) with the FDR that is actually achieved by the tool according to the ground truth. (The line for QPROT is not shown as it is lierally off the scale.) B) Plot showing how many actually changed proteins (true positives) each method identified at a specified FDR level. The methods shown as dotted lines should be considered "disqualified" as they that failed to control the FDR in panel A. 


\section{Supplementary Files}

This is a list of supplementary files associated with this preprint. Click to download.

- proDAappendicies.pdf 